\title{
Effect of educational intervention on pressure ulcer documentation among nurses in Jordan Ministry of Health Tertiary Hospitals: a quasiexperimental research approach
}

\begin{abstract}
Background: Pressure ulcers (PUs) are areas of localised tissue destruction that occur as a soft tissue gets compressed over a bony prominence and an external skin surface for a prolonged period of time. Treatment of ulcers of the sort represents a major financial burden for most health care systems worldwide as well as in Jordan and Arab world. Objective: the aim of this study is to develop, implement and evaluate the effect of intervention educational on nursing quality of documentation regarding pressure ulcer in Jordanian ministry of health tertiary hospitals. Methods/design: A quasi experimental study design will be conducted in Jordan in which 54 nurses will be placed in each of two groups, a control and an intervention group. Nurses will be selected to represent the following four wards: (1) the surgical ward, (2) the medical ward, (3) the intensive care unit and (4) the orthopaedic ward. Sampling will consider the number of nurses in each of those wards. Stratification and a simple random sampling technique will be used to sample 54 nurses in each group. Pre- and postintervention assessments will be conducted to evaluate two parameters with respect to: (1) knowledge, skills, motivation and (2) quality of nursing documentation. The quality of documentation regarding pressure ulcer will be determined by examining two documents for each participant every time using the nursing documentation Chart Audit Tool. One-way multivariate analysis of variance and 2-way repeated multivariate analysis of variance will be used to analyze the data. Discussion: To the best of our knowledge, this research will be the first quasi experimental time series study with control group using a lectures educational intervention for nurses to examine the quality of nursing documentation on pressure ulcer, knowledge, skills, and motivation regarding nurses.
\end{abstract}

Keyword: Intervention; Pressure ulcer (PU); Information Motivation Behavioral Skills Model; Quality of nursing documentation; Quasi experimental 